Review

\title{
Specific Markers for Hepatic Progenitor Cells
}

\author{
${ }^{1,2,4}$ Msy Rulan Adnindya, ${ }^{1,2,5}$ Indriyani, ${ }^{1,2,6}$ Ahmad Azmi Nasution, ${ }^{1,2,3}$ Isabella Kurnia Liem \\ ${ }^{I}$ Department of Anatomy, Faculty of Medicine, Universitas Indonesia, Jakarta, Indonesia \\ ${ }^{2}$ Master Program in Biomedical Sciences, Faculty of Medicine, Universitas Indonesia, Jakarta, Indonesia \\ ${ }^{3}$ Integrated Laboratory, Faculty of Medicine, Universitas Indonesia, Indonesia \\ ${ }^{4}$ Department of Anatomy, Faculty of Medicine, Sriwijaya University, Palembang, Indonesia \\ ${ }^{5}$ Department of Anatomy, Faculty of Medicine, Universitas Muhammadiyah Palembang, Palembang, Indonesia \\ ${ }^{6}$ Department of Anatomy, Faculty of Medicine and Health Science, Universitas Bengkulu, Bengkulu, Indonesia
}

Article history

Received: 28-12-2016

Revised: 09-03-2017

Accepted: 12-07-2017

Corresponding Author: Isabella Kurnia Liem

Department of Anatomy,

Faculty of Medicine,

Universitas Indonesia, Jakarta,

Indonesia

Email: bellajo04@gmail.com

isabella.kurnia@ui.ac.id

\begin{abstract}
One of mammalian organs that has remarkable ability to regenerate, liver, regenerates through the proliferation of hepatocytes or proliferation and differentiation of the stem cells resident. Some literatures states that these stem cells, the Hepatic Progenitor Cells (HPCs), are oval cells that have bipotential ability to be cholangiocytes and hepatocytes. However, the mechanisms of programming, activation of proliferation and maturation/differentiation have not completely understood. There are still disagreements on the sources and characterictics of HPCs. Consequently, these lead to a question on the characteristic (specific marker) of HPCs. To summarize the specific markers for HPCs that have ever been identified, the writers did a systematic review on 12 papers that fulfilled the research criteria and concluded that the markers used to identify HPCs are a combination of classic HPCs markers, i.e., oval cells (OV6 and OC2) progenitor/immature cells (CD56, Oct-3/4, DLK1, EFNA1 and Chrom-A), hepatocytes and cholangiocytes marker (Alb, AFP, CK7, CK8, CK18, CK19, GGT, EpCAM, CD24 and CD44) and hematopoietic stem cells markers (CD34, CD45, CD109, CD133, c-kit and Thy-1) since HPCs may share similar lineage as hematopoietic stem cells, hepatocytes and cholangiocytes. Precise markers for HPCs could not yet be determined; therefore, several markers should be combined to identify the HPCs. Difficulties in finding precise markers for HPCs might be caused by different expression of markers based on the HPCs differentiation and maturity.
\end{abstract}

Keywords: Hepatic Progenitor Cells, Hepatocyte, Hepatoblast, Oval Cells, Cholangiocyte, Hepatic Progenitor Cells Marker

\section{Introduction}

Liver has an outstanding ability to regenerate itself through hepatocytes proliferation. The uniqueness of liver regeneration is that the responsible cells to repair the liver are differentiated cell, i.e., hepatocytes and cholangiocytes, despite of its low of mitotic rate (Hu et al., 2007; Marongiu et al., 2014). Nevertheless, the responsibility to restore liver mass is taken over by Hepatic Progenitor Cells (HPCs) under certain condition that deteriorates hepatocytes, such as in chronic liver pathologic condition or severe loss of liver mass $(70 \%$ hepatectomy) (Mavila et al., 2014; Fausto, 2004). The HPCs are assumed to be an embryonic residual from embryonic hepatoblast that remain in adult liver; even though, the origin and relationship between them have not yet been clearly understood. These cells are described as oval shaped cells with oval nucleus and high cytoplasmic nuclear ratio, which is also known as oval cells (Hu et al., 2007).

Oval cells are bi-potent as they can differentiate into hepatocytes (positive for Albumin and positive or negative for AFP) and cholangiocytes (positive for CK19), characteristics that are similar to embryonic hepatoblast (Zhou et al., 2007; Mavila et al., 2014; Hu et al., 2007; Romano et al., 2015). The cells serve as hepatic progenitor cells in adult liver and display a highly proliferative activity during chronic liver injury. They were found both in rodent and human at Canal of Hering alongside the portal triad (Mavila et al., 2014; Hu et al., 
2007). Proliferation of oval cells starts after liver injury or when hepatocyte function is impaired, as displayed in animal model induced with 2-Acetylaminofluore Followed with Partial Hepatectomy (2-AAF/PH). In the model, proliferation of hepatocyte was inhibited by 2AAF; consequently, oval cells took the responsibility to repair the liver after losing its mass by partial hepatectomy (Paku et al., 2004; Darwiche et al., 2011).

Oval cells were also known to have proliferative response to carcinogenic agents, although it has not been proven yet whether oval cells have a role as cancer progenitor cells. The activated hepatic progenitor cells were expressed protein markers for progenitor/stem cells, i.e. EpCAM, OV6, Prominin 1, CD49f and A6, with AFP positive or negative. In this condition, however, similar to the maturation and differentiation process, it has not been agreed whether there are any reprogramming of hepatocytes or not (Mavila et al., 2014). In liver malignancy and chronic liver disease, HPCs presented as Ductular Reactions (DR). Ductular reactions is a response to chronic liver injury hence HPC in Canal of Hering proliferate and form irregular shaped structure existed in parenchymal-stromal boundaries (Hu et al., 2007; Fausto, 2004; Zhou et al., 2007). Using specific-lineage tracing system, Jors et al. (2015) found that in its regeneration, biliary cells did not originate from HPCs, but derived from adult biliary compartment. This study implies that biliary progenitor cells (ductular reactive cells/DRCs) do not have bipotential ability.

There are disagreements on the sources and characterictics of HPCs, since their mechanisms of programming, activation of proliferation and maturation/differentiation have not yet been completely understood. It is still a question, why oval cells can only be indentified in the event of a severe liver injury. Consequently, specific markers are crucial in identifying hepatic progenitor cells. Recently, many antibodies have been developed to provide specific markers for HPCs; however, these markers have not yet been generally accepted. Therefore, a systematic review aimed to explore various existing markers for hepatic progenitor cells and their locations in liver tissues has been done.

\section{Methods}

Published researches were collected by searching original research articles in Pubmed/Medline, Pubmed Central and Cochrane library, while unpublished research were obtained from database of Universitas Indonesia library (http://www.lib.ui.ac.id). "Oval cell" OR "hepatic progenitor cell" AND "marker" AND 'liver" OR "hepar" were used as keywords. The searching time was unlimited. Articles from writer's libraries were also added. Inclusion criteria and exclusion criteria were applied in this study. Inclusion criteria were for all studies that identified oval cells, used immunohistochemistry staining on $2 \mathrm{AAF} / \mathrm{PH}$ rat animal model for oval cell or liver malignancy (e.g., hepatocelullar carcinoma and hepatoblastoma) tissue. Meanwhile, the exclusion criteria were for non English written articles and in vitro studies. Collected data were markers that were used to identify the oval cells and the location of positive-stain oval cells. Data were noted and tabulated.

\section{Results}

From the searching on November 23, 2016, 59 articles were collected. Majority of the articles were in vitro studies; however, some of them did not investigate $\mathrm{HPCs}$ in liver, $2 \mathrm{AAF} / \mathrm{PH}$ rat animal model for liver injury, or liver malignancy. After the writers ruled out articles that did not meet the inclusion criteria, there were only 12 articles left to be studied.

Markers that were used to identify Hepatic Progenitor/oval Cells (HPCs) in previous studies are presented in Table 1. It can be seen that a total of 23 different markers were used to identify HPCs. They are markers for hepatocytes and cholangiocytes (Alb, AFP, CK7, CK8, CK18, CK19, GGT, EpCAM, CD24 and CD44), hematopoietic stem cells (CD34, CD45, CD109, CD133, c-kit and Thy-1) and classic markers for oval cells and progenitor/immature cells (OV6, OC2, CD56, Oct-3/4, DLK1, EFNA1 and Chrom-A). In order to identify HPCs, combination of several markers was used.

HPCs have bipotential ability to differentiate into both hepatocytes and cholangiocytes. In Table 2, expression of markers among hepatocytes, cholangiocytes and HPCs were compared. HPCs have common markers with hepatocytes and cholangiocytes but the expression is not precisely similar. Some inconsistencies were found such as in expression of AFP, CK7 and c-kit.

Distribution expression of each marker can be seen in Table 3. HPCs are detected in Canal of Hering, periportal region, bile epithelial duct, bile ductular cells and single oval cell-like cells.

\section{Discussion}

Many studies used several markers to identify hepatic progenitor cells. Several markers for Hematopoietic Stem Cells (HSCs) have been proposed since those markers positive cells were suspected as the origin of hepatic progenitor cells. Li et al. (2014) used 10 markers to identify HPCs; eight markers for HPCs (Alb, AFP, CK8, CK18, CK19, CD90, CD117 and OV6) and two markers for HSCs (CD45 and CD109). The similarity of HPCs and hepatocytes were confirmed. HPCs also express cholangiocytes markers (CK19) moderately and HSCs marker (CD45 and CD109) strongly. 
Table 1. Reported markers for hepatic progenitor/oval cell

\section{Reference}

Akiba et al. (2013)

Badrawy et al. (2013)

Chen et al. (2015)

Hao et al. (2013)

Jia et al. (2013)

Li et al. (2014)

Libbrecht et al. (2000)

Petersen et al. (1998)

Van Den Heuvel et al. (2001)

Ye et al. (2014)

Yun et al. (2013)

Yovchev et al. (2007)
Positive-stained

CD56, c-kit (CD117), CD133, EpCAM

OV6, AFP

OV6, CK19

EpCAM, CD133, AFP, EFNA1

OV6

Alb, AFP, CK8, CK18, CK19, CD90, c-kit (CD117), OV6, CD45, CD109

CK7, CK19, CK8, CK18, Chrom-A, OV6

AFP, GGT, OV6, CK19, OC2, Thy-1

OV6, CK19

EpCAM, OV6, CD133, c-kit (CD117)

CK19, Oct-3/4, EpCAM, DLK1

EpCAM, CD44, CD24, CD133

Table 2. Comparison of markers in hepatocytes, cholangiocytes and hepatic progenitor cells

\begin{tabular}{|c|c|c|c|}
\hline & Hepatocytes & Cholangiocytes & HPCs \\
\hline OV6 & - & + & + \\
\hline CD45 & ++ & ND & ++ \\
\hline CD109 & ++ & ND & ++ \\
\hline CK8 & + & ND & ++ \\
\hline CK18 & + & ND & ++ \\
\hline AFP & - & - & $+/-$ \\
\hline Albumin & + & - & + \\
\hline CK7 & $+/-$ & + & + \\
\hline CK19 & - & + & ++ \\
\hline CD34 & ND & ND & + \\
\hline Oct-3/4 & ND & ND & + \\
\hline EpCAM & - & + & + \\
\hline c-Kit (CD117) & - & - & $+/-$ \\
\hline CD133 & + & - & + \\
\hline Thy-1 & - & - & + \\
\hline Dlk1 & - & - & + \\
\hline Chrom-A & - & ND & + \\
\hline
\end{tabular}

$\mathrm{ND}=$ Not Determined; +/- = positive and negative

Table 3. Location of positive-stained marker for hepatic progenitor cell

\begin{tabular}{|c|c|c|}
\hline Reference & Marker & Location of HPCs \\
\hline \multirow[t]{2}{*}{ Badrawy et al. (2013) } & OV6 & Scattered bile duct lining cells in cirhosis hepatis \\
\hline & AFP & Bile ducts or hepatic parenchyme adjacent to the duct \\
\hline Chen et al. (2015) & OV6, CK19 & $\begin{array}{l}\text { Fibrous septum, duct-like structures surrounding the portal tract } \\
\text { and in fibrotic septa }\end{array}$ \\
\hline Jia et al. (2013) & OV6 & $\begin{array}{l}\text { Sinusoidal pole of hepatocytes, hepatic cord and bile duct cell in } \\
\text { the portal tract }\end{array}$ \\
\hline Li et al. (2014) & $\begin{array}{l}\text { OV6, CK8, CK18, CK19, } \\
\text { CD90, CD117, CD45, CD109 }\end{array}$ & Canal of Hering and periportal areas \\
\hline Van Den Heuvel et al. (2001) & OV6, CK19 & $\begin{array}{l}\text { Bile epithelial duct, bile ductular cell, canal of Hering, single oval } \\
\text { cell-like cells located at the interface between portal mesenchyme } \\
\text { and hepatic parenchyma }\end{array}$ \\
\hline \multirow[t]{3}{*}{ Ye et al. (2014) } & EpCAM & Cholangiocytes (canal of Hering, ductules and small and large bile duct) \\
\hline & OV6 & Adjacent to duct of marginal region \\
\hline & CD133 & A few scattered hepatocyte \\
\hline
\end{tabular}

In addition, Ye et al. (2014) used four markers for HPCs (EpCAM, OV6, CD133 and c-kit) and found that HPCs express HSCs marker (c-kit and CD133) and express biliary marker (EpCAM). Another HSCs marker used to identify HPCs was Thy-1 (Petersen et al., 1998).
In further, Yovchev et al. (2007) found that oval cells/HPCs express CD44, CD24, CD133 and EpCAM. CD44, CD24 and EpCAM were expressed in HPCs and biliary epithelial cells. During embryonic period, the first main hematopoietic site is liver. It is assumed that there is a trace of HSCs left in the adult liver (Petersen et al., 
1998). Thus, HPCs possibly will express the same markers as HSCs. By comparing trancription factors in HPCs and HSCs, different expressions in both cells were observed (Chen et al., 2013). Therefore, it is possible that HPCs and HSCs share similar origin but are differentiated into different lineage.

Meanwhile, oval cells in the adult liver express both hepatocyte and cholangiocyte markers such as albumin, GGT, CK7, CK19 and EpCAM (Ye et al., 2014; Petersen et al., 1998). HPCs express CK19 which is positive in cholangiocytes but undetectable in hepatocytes (Yun et al., 2013). Another study suggested that HPCs express the same marker as primitive bile duct cells of the ductal plate, AFP, but have different phenotypic features from cholangiocytes and hepatocytes. HPCs characteristic were in the middle between those of stem cells and mature cells (Van Den Heuvel et al., 2001; Petersen et al., 1998; Li et al., 2014; Hao et al., 2013). This finding suggested that HPCs have bipotential capacity to differentiate into both cholangiocytes and hepatocytes. HPCs also express the same markers as hepatoblast, such as DLK1; even though, there has not been an agreement for AFP expression. HPCs also express stem cells markers such as oct-3/4 (Yun et al., 2013). Oct-3/4 is expressed in cells that have pluripotency and has important role in maintaining the pluripotency (Okumura-nakanishi et al., 2005). This implied that HPCs are young, developing cells (Yun et al., 2013).

In identifying HPCs, various markers are used. Jia et al. (2013) identified oval cells in HCC using classic marker for HPCs, i.e., OV6 antibody. OV6 positive cells were found in some locations, such as in hepatic cords, undifferentiated cell at the sinusoidal end of hepatocytes and in bile ductal cells in the portal tract. Similarly, Ye et al. (2014) found that OV6positive cells similar to the oval cells adjacent to ducts. Co-expression between OV6 and CK19 were also observed. These findings support the hipothesis that oval cells and cholangiocytes derived from the same lineage. The positive expression a were found in a normal liver and an injured liver. Because OV6 identifies HPCs in normal and injured liver, OV6 marker cannot distinguish between normal and active cells (Van Den Heuvel et al., 2001).

In further, Badrawy et al. (2013) used OV6 and AFP to detect HPCs, since AFP can be used as a sign for an initial hepatic lineage and stem cell activation. Another marker that is also possible to be used as oval cells' marker is CD56. The expressions of CD56 indicate regenerative condition of liver cells and were found in ductular cells and oval cells (Van Den Heuvel et al., 2001; Akiba et al., 2013)). OC2 is the other traditional marker that can be used for oval cells. Study shows that oval cells express both OC2 and OV6 were located in periportal (Petersen et al., 1998; Faris et al., 1991).

Meanwhile, EFNA1 is over expressed in tumor cells, i.e., HCC and related to endothelial cell migration, tumor progression, angiogenesis (Cui et al., 2010). Hao et al. (2013) found expression of EFNA1 in ductular reaction is correlated to AFP. EFNA1 expressions were stronger than AFP, thus EFNA1 can be used as HPCs.

The other marker that can be used to identify HPCs is Chromogranin-A. Libbrecht et al. (2000) defined progenitor cells in human and rat liver as small cells that express CK7, CK19, CK8, CK18, OV6 and chrom-A.

HPCs share similar markers with hepatocytes and cholangiocytes, but not identically. It is assumed that it is because of the bipotential capacity of HPCs to differentiate into both cells. The data also shows inconsistency of some marker expressions such as AFP and c-kit in HPCs that needed to be investigated further. Various expressions of HPCs imply that the population of progenitor cells are consisted of heterogenous cells or cells with different maturity.

HPCs are detected in Canal of Hering, periportal region, bile epithelial duct, bile ductular cells and single oval cell-like cells. HPCs normally exist in a periportal region are consisted of heterogenous cell population. In the beginning of regeneration, HPCs are discovered in the proximal branches of the biliary tree, comprising the bile ductules and Canal of Hering.

\section{Conclusion}

This review describes a number of markers used to identify hepatic progenitor/oval cells and the distribution of markers expression in the liver. Markers that were used to identify hepatic progenitor/oval cells are a combination of classic HPCs markers (OV6 andOC2), other HPCs markers (EFNA1, Chrom-A, CD56 and Oct$3 / 4$ ), hepatocytes and cholangiocytes marker (Alb, AFP, CK7, CK8, CK18, CK19, GGT, EpCAM, CD24 and CD44) and hematopoietic stem cells markers (CD34, CD45, CD109, CD133, c-kit and Thy-1),because HPCs may share similar lineage as HSCs, hepatocytes and cholangiocytes. The reason for various expressions found in different studies of hepatic progenitor/oval cells might be caused by different expression based on the HPCs differentiation and maturity. Even though, the roles of HPCs are important for liver regeneration, but researchers have not yet agreed about the precise marker for HPCs. The precise marker for HPCs will provide better information of HPCs' characteristics. Therefore, precise marker for HPC is needed to be investigated in further by investigating each marker's expression and/or its co-expression during liver development and in specific time and condition that triggers proliferation of HPCs (i.e., liver injury or carcinoma). 


\section{Acknowledgement}

Our gratitude is extended to Department of Anatomy, Faculty of Medicine, Universitas Indonesia for facilitating and supporting this study.

\section{Funding Information}

The review was funded by the grant from the Directorate Research and Community Engangement Universitas Indonesia, Hibah Publikasi Internasional Terindeks untuk Tugas Akhir Mahasiswa UI 2016 with the contract number of 1916/UN2.R12/HKP.05.00/2016.

\section{Author's Contributions}

Each author has an equal contribution in the preparation, development and publication of this manuscript.

\section{Ethics}

This review does not have any ethical problems since the subject was published original articles.

\section{References}

Akiba, J., O. Nakashima, S. Hattori, K. Tanikawa and M. Takenaka et al., 2013. Clinicopathologic analysis of combined hepatocellular-cholangiocarcinoma according to the latest WHO classification. Am. J. Surgical Pathol., 37: 496-505.

DOI: $10.1097 /$ PAS.0b013e31827332b0

Badrawy, N.E., O.A. Hammam, M.E. Ghanam, M.A. Ansary and M. Hassan et al., 2013. OV6, $\alpha$ fetoprotein, hepatocyte growth factor and transforming growth factor beta 1 in patients with chronic hepatitis, cirrhosis and heap to cellular carcinoma. J. Am. Sci., 9:647-657.

Chen, J., X. Zhang, Y. Xu, X. Li and S. Ren et al., 2015. Hepatic progenitor cells contribute to the progression of 2-acetylaminofluorene/carbon tetrachloride-induced cirrhosis via the non-canonical Wnt pathway. PLoS One, 10:1-17.

DOI: 10.1371 journal.pone. 0130310

Chen, Q., M. Khoury, G. Limmon, M. Choolani and J.K.Y. Chan et al., 2013. Human fetal hepatic progenitor cells are distinct from, but closely related to, hematopoietic stem/progenitor cells. Stem Cells, 31: 1160-1169.

DOI: $10.1002 /$ stem. 1359

Cui, X., M. Lee, G. Yu, I. Kim and H. Yu et al., 2010. EFNA1 ligand and its receptor EphA2: Potential biomarkers for hepatocelullar carcinoma. Int. J. Cancer, 126: 940-949. DOI: 10.1002/ijc.24798
Darwiche, H., S. Oh, N.C. Steiger-Luther, J.M. Williams and D.G. Pintilie et al., 2011. Inhibition of notch signaling affects hepatic oval cell response in rat model of 2AAF-PH. Hepatic Med., 3: 89-98. DOI: 10.2147/HMER.S12368

Fausto, N., 2004. Liver regeneration and repair: Hepatocytes, progenitor cells and stem cells. Hepatology, 39: 1477-1487.

DOI: $10.1002 /$ hep. 20214

Faris, R.A., B.A. Monfils, H.A. Dunsford and D.C. Hixson, 1991. Antigenic relationship between oval cells and a subpopulation of hepatic foci, nodules and carcinomas induced by the "resistant hepatocyte" model system. Cancer Res., 51: 1308-1317.

PMID: 1997169

Hao, P., M. Lee, G. Yu, I. Kim and Y. Cho et al., 2013. Isolation of EpCAM+/CD133- hepatic progenitor cells. Molecules Cells, 36:424-431. DOI: $10.1007 / \mathrm{s} 10059-013-0190-\mathrm{y}$

$\mathrm{Hu}$, M., M. Kurobe, Y.J. Jeong, C. Fuerer and S. Ghole et al., 2007. Wnt/ $\beta$-catenin signaling in murine hepatic transit amplifying progenitor cells. Gastroenterology, 133: 1579-1591. DOI: $10.1053 /$ j.gastro.2007.08.036

Jia, S., J. Ren, P. Dong and X. Meng, 2013. Probing the hepatic progenitor cell in human hepatocellular carcinoma. Gastroenterol Res. Pract. DOI: $10.1155 / 2013 / 145253$

Jors, S., P. Jeliazkova, M. Ringelhan, J. Thalhammer and S. Dürl et al., 2015. Lineage fate of ductular reactions in liver injury and carcinogenesis. J. Clin. Invest., 125: 2445-2457. DOI: 10.1172/JCI78585

Li, J., J. Xin, L. Zhang, J. Wu and L. Jiang et al., 2014. Human hepatic progenitor cells express hematopoietic cell markers CD45 and CD109. Int. J. Med. Sci., 11:65-79. DOI: 10.7150/ijms. 7426

Libbrecht, L., V. Desmet, B.V. Damme and T. Roskams, 2000. The immunohistochemical phenotype of dysplastic foci in human liver: Correlation with putative progenitor cells. J. Hepatol., 33: 76-84. DOI: $10.1016 / \mathrm{S} 0168-8278(00) 80162-2$

Marongiu, F., M.P. Serra, M. Sini, M. Marongiu and A. Contini et al., 2014. Cell turnover in the repopulated rat liver: Distinct lineages for hepatocytes and the biliary epithelium. Cell Tissue Res., 356: 333-340. DOI: 10.1007/s004414-1800-5

Mavila, N., M.V. Nguyen, D. James and K.S. Wang, 2014. Progenitor cells in liver development, regeneration and repair. Cell Dev. Biol. DOI: $10.4172 / 2168-9296.1000144$

Okumura-Nakanishi, S., M. Saito, H. Niwa and F. Ishikawa, 2005. Oct-3/4 and Sox2 regulate Oct$3 / 4$ gene in embryonic stem cells. J. Biol. Chem., 280: 5307-5317. DOI: 10.1074/jbc.M410015200 
Paku, S., P. Nagy, L. Kopper and S.S. Thorgeirsson, 2004. 2-Acetylaminofluorene dose-dependent differentiation of rat oval cells into hepatocytes: Confocal and electron microscopic studies. Hepatology, 39: 1353-1361. DOI: $10.1002 /$ hep. 20178

Petersen, B.E., J.P. Goff, J.S. Greenberger and G.K. Michalopoulos, 1998. Hepatic oval cells express the hematopoietic stem cell marker Thy-1 in the rat. Hepatology, 27: 433-445. DOI: $10.1002 /$ hep.510270218

Romano, M., F.D. Francesco, G. Pirozzi, E. Gringeri and R. Boetto et al., 2015. Expression of cancer stem cell biomarkers as a tool for a correct therapeutic approach to hepatocellular carcinoma. Oncoscience, 2: 443-456.

Van Den Heuvel, M.C., M.J.H. Slooff, L. Visser, M. Muller and K.P. De Jong et al., 2001. Expression of anti-OV6 antibody and anti-N-CAM antibody along the biliary line of normal and diseased human livers. Hepatology, 33: 1387-1393.

DOI: $10.1053 /$ jhep.2001.24453
Ye, F., Y. Jing, S. Guo, G. Yu and Q. Fan et al., 2014. Proliferative ductular reactions correlate with hepatic progenitor cell and predict recurrence in HCC patients after curative resection. Cell Biosci., $4: 1-14$.

Yun, W.J., E. Shin, K. Lee, H.Y. Jung and S.H. Kim et al., 2013. Clinicopathologic implication of hepatic progenitor cell marker expression in hepatoblastoma. Pathol. Res. Pract., 209:568-573. DOI: 10.1016/j.prp.2013.06.015

Yovchev, M.I., P.N. Grozdanov, B. Joseph, S. Gupta and M.D. Dabeva, 2007. Novel hepatic progenitor cell surface markers in the adult rat liver. Hepatology, 45: 139-148. DOI: 10.1002/hep. 21448

Zhou, H., L.E. Rogler, L. Teperman, G. Morgan and C.E. Rogler, 2007. Identification of hepatocytic and bile ductular cell lineages and candidate stem cells in bipolar ductular reactions in ccirrhotic human liver. Hepatology, 45: 716-724.

DOI: $10.1002 /$ hep. 21557 